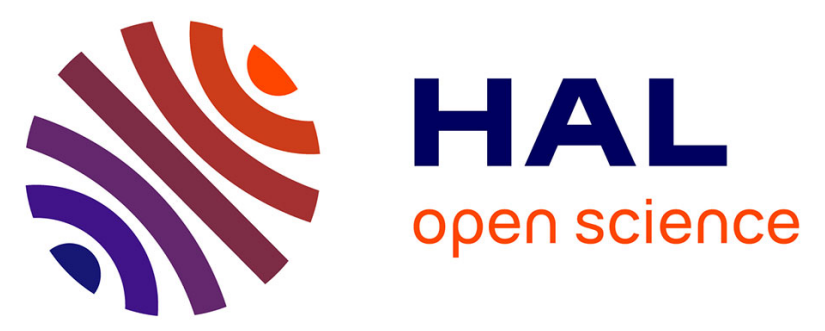

\title{
Heavy Ion Induced Degradation in SiC Schottky Diodes: Incident Angle and Energy Deposition Dependence
}

\author{
Arto Javanainen, Marek Turowski, Kenneth Galloway, Christopher Nicklaw, \\ Véronique Ferlet-Cavrois, Alexandre Louis Bosser, Jean-Marie Lauenstein, \\ Michele Muschitiello, Francesco Pintacuda, Robert Reed, et al.
}

\section{To cite this version:}

Arto Javanainen, Marek Turowski, Kenneth Galloway, Christopher Nicklaw, Véronique FerletCavrois, et al.. Heavy Ion Induced Degradation in SiC Schottky Diodes: Incident Angle and Energy Deposition Dependence. IEEE Transactions on Nuclear Science, 2017, 64 (8), pp.2031-2037. 10.1109/TNS.2017.2717045 . lirmm-02021535

\section{HAL Id: lirmm-02021535 \\ https://hal-lirmm.ccsd.cnrs.fr/lirmm-02021535}

Submitted on 20 Dec 2019

HAL is a multi-disciplinary open access archive for the deposit and dissemination of scientific research documents, whether they are published or not. The documents may come from teaching and research institutions in France or abroad, or from public or private research centers.
L'archive ouverte pluridisciplinaire HAL, est destinée au dépôt et à la diffusion de documents scientifiques de niveau recherche, publiés ou non, émanant des établissements d'enseignement et de recherche français ou étrangers, des laboratoires publics ou privés. 
This is an electronic reprint of the original article. This reprint may differ from the original in pagination and typographic detail.

\author{
Author(s): Javanainen, Arto; Turowski, Marek; Galloway, Kenneth F.; Nicklaw, Christopher; \\ Ferlet-Cavrois, Véronique; Bosser, Alexandre; Lauenstein, Jean-Marie; Muschitiello, \\ Michele; Pintacuda, Francesco; Reed, Robert A.; Schrimpf, Ronal D.; Weller, Robert A.; \\ Virtanen, Ari
}

Title: Heavy-Ion-Induced Degradation in SiC Schottky Diodes : Incident Angle and Energy Deposition Dependence

Year: $\quad 2017$

Version:

Please cite the original version:

Javanainen, A., Turowski, M., Galloway, K. F., Nicklaw, C., Ferlet-Cavrois, V., Bosser, A., ... Virtanen, A. (2017). Heavy-Ion-Induced Degradation in SiC Schottky Diodes : Incident Angle and Energy Deposition Dependence. IEEE Transactions on Nuclear Science, 64 (8), 2031-2037. doi:10.1109/TNS.2017.2717045

All material supplied via JYX is protected by copyright and other intellectual property rights, and duplication or sale of all or part of any of the repository collections is not permitted, except that material may be duplicated by you for your research use or educational purposes in electronic or print form. You must obtain permission for any other use. Electronic or print copies may not be offered, whether for sale or otherwise to anyone who is not an authorised user. 


\title{
Heavy Ion Induced Degradation in $\mathrm{SiC}$ Schottky Diodes: Incident Angle and Energy Deposition Dependence
}

\author{
Arto Javanainen, Member, IEEE, Marek Turowski, Senior Member, IEEE, \\ Kenneth F. Galloway, Fellow, IEEE, Christopher Nicklaw, Véronique Ferlet-Cavrois, \\ Fellow, IEEE, Alexandre Bosser, Student Member, IEEE, Jean-Marie Lauenstein, Member, \\ IEEE, Michele Muschitiello, Francesco Pintacuda, Robert A. Reed, Fellow, IEEE, Ronald \\ D. Schrimpf, Fellow, IEEE, Robert A. Weller, Senior Member, IEEE, and A. Virtanen, \\ Member, IEEE
}

\begin{abstract}
Heavy-ion induced degradation in the reverse leakage current of SiC Schottky power diodes exhibits a strong dependence on the ion angle of incidence. This effect is studied experimentally for several different bias voltages applied during heavy-ion exposure. Additionally, TCAD simulations are used to give insight on the physical mechanisms involved.
\end{abstract}

Index Terms - Ion radiation effects, Modeling, Power semiconductor devices, Schottky diodes, Silicon carbide

\section{INTRODUCTION}

$\mathrm{S}$ ILICON CARBIDE ( $\mathrm{SiC}$ ) has very good properties for use in power device applications. In comparison to silicon, it has higher breakdown field and higher thermal conductivity. However, the sensitivity of $\mathrm{SiC}$ power devices (MOSFETs and diodes) to particle radiation has

This work was supported by the Walter Ahlström Foundation through the Tutkijat Maailmalle program, the European Space Agency (ESA/ESTEC Contract 4000111630/14/NL/PA), and the Academy of Finland under the Finnish Centre of Excellence Programme 2012-2017 (Project No 2513553, Nuclear and Accelerator Based Physics).

A. Javanainen, A. Bosser and A. Virtanen are with the University of Jyvaskyla, Department of Physics, P.O. Box 35, FI-40014, University of Jyvaskyla, Finland. (email: \{arto.javanainen, alexander.l.bosser, ari.j.virtanen\}@jyu.fi)

A. Javanainen is also with the Electrical Engineering and Computer Science Department, Vanderbilt University, Nashville, TN 37235 USA. (email: arto.javanainen@vanderbilt.edu)

A. Bosser is also with LIRMM, University of Montpellier/CNRS, France.

K. Galloway, R. Reed, R. Schrimpf and R. Weller are with the Electrical Engineering and Computer Science Department, Vanderbilt University, Nashville, TN 37235 USA. (email: \{kenneth.f.galloway, robert.reed, ron.schrimpf, robert.a.weller\}@ vanderbilt.edu)

C. Nicklaw and M. Turowski are with Silvaco Inc. 4701 Patrick Henry Drive, Bldg. 2, Santa Clara, CA 95054, USA, (email: \{chris.nicklaw, marek.turowski\}@silvaco.com)

V. Ferlet-Cavrois and M. Muschitiello are with the European Space Agency, ESTEC, 2200 AG Noordwijk, The Netherlands. (email: veronique.ferlet-cavrois@esa.int)

J. M. Lauenstein is with NASA/GSFC, Code 561.4, Greenbelt, MD 20771 USA, (email: jean.m.lauenstein@nasa.gov)

F. Pintacuda is with the STMicroelectronics Srl, I-95121 Catania, Italy. (email: francesco.pintacuda@ st.com) been found to be surprisingly high [1]-[3]. Moreover, the radiation response of $\mathrm{SiC}$ Schottky devices to heavy ions has been shown to differ from that of silicon-based power devices. The silicon power devices typically exhibit separate regions of destructive and nondestructive response as a function of voltage [4], [5]. In addition to these two regions, SiC Schottky devices have been reported to exhibit also a third region where devices suffer from gradual degradation under heavy-ion exposure [2], [6], [7]. This gradual degradation complicates the assessment of $\mathrm{SiC}$ parts for catastrophic failures like Single Event Burnout (SEB). Typically, these parts are tested by using heavy-ion beams perpendicular to the device surface. For most silicon power devices, this is considered to be the worst-case exposure for destructive failures, depending on the actual device geometry [8].

In this paper, we show that a heavy-ion beam at normal incidence is also the worst case for SiC Schottky power diodes. The degradation rates exhibit significant reduction with increasing tilting angle for these devices at constant reverse-bias. The behavior does not follow any typical cosine-laws. The observed degradation has been attributed to thermal effects that are initiated by complex interactions between the reverse-bias applied to the diode and the energy deposited by the incident heavy-ions [9], [10].

In this work, TCAD simulations have been performed to study the thermo-electric response of SiC Schottky structure to heavy ions. The simulation results are consistent with the explanation that the differences in the degradation rates observed at different tilting angles are due to different thermal responses. A simplified explanation is that the misalignment between the ioninduced charge filament and the potential gradient 
within the diode depletion region reduces the density of the collected charge within the Schottky structure, hence the degradation.

This paper builds on the work presented at the RADECS 2016 conference and partly reported in [11].

\section{EXPERIMENTAL METHODS}

The devices used in this study were commercial SiC Schottky power diodes, manufactured by STMicroelectronics. The device types were STPSC1006D (600V, 10A) and STPSC10H065DY $(650 \mathrm{~V}, 10 \mathrm{~A})$. The first device type is based on a plain Schottky junction structure, and the latter is a junction barrier Schottky (JBS) diode. The epilayer thickness in both diodes is about $6 \mu \mathrm{m}$.

The plastic packaging material was removed from the diodes prior to irradiation to enable sufficient penetration for the heavy ions inside the device, i.e., beyond the Schottky junction and the epitaxial layer. The total thickness of overlying passivation layers and the epitaxial layer was estimated to be on the order of ten micrometers.

The heavy-ion irradiations for this work were performed at the RADiation Effects Facility (RADEF) in the Accelerator Laboratory of the University of Jyväskylä [12]. This paper presents results obtained for $\mathrm{Xe}$ ions with energy of $1217 \mathrm{MeV}$. The linear energy transfer (LET) value for these ions is $62 \mathrm{MeV} /\left(\mathrm{mg} / \mathrm{cm}^{2}\right)$ and the average projected range in $\mathrm{SiC}$ is $60 \mu \mathrm{m}$, as estimated by using the code from [13]. All the irradiations and the device characterization were performed at ambient temperature in vacuum conditions. The incident angle of the ion beams relative to normal was varied between 0 and 45 degrees. The ion flux ranged between 20 and $6 \cdot 10^{5} \mathrm{~cm}^{-2} \cdot \mathrm{s}^{-1}$ and cumulative fluences between the irradiation runs varied from $10^{3}$ to $10^{6} \mathrm{~cm}^{-2}$.

The current-voltage characteristics for the devices were measured both for the forward and the reverse voltages, before and after each irradiation run. During the irradiations, the device under test (DUT) was biased at fixed reverse voltage and the leakage current was monitored. The device biasing and characterization was done using a Keithley 2410 Source Measure Unit [14]. The voltage limit for the 2410 is $1100 \mathrm{~V}$. Because the DUTs were not coated, the maximum voltage levels during testing were limited to about $-350 \mathrm{~V}$ due to arcing that occurs approximately at $-400 \mathrm{~V}$.

\section{EXPERIMENTAL RESULTS}

Heavy-ion exposure has been shown to increase reverse leakage current in SiC Schottky diodes, when a sufficient reverse bias is applied during radiation exposure [6], [7], [15]. When an ion beam hits the device at an angle off normal incidence, a similar, but weaker, effect is observed [11]. This is illustrated in Figure 1, where the evolution of leakage current for an STPSC10H065DY diode during Xe-ion exposure is given for three different biasing configurations, while the device was tilted at $20^{\circ}$ off from normal incidence.

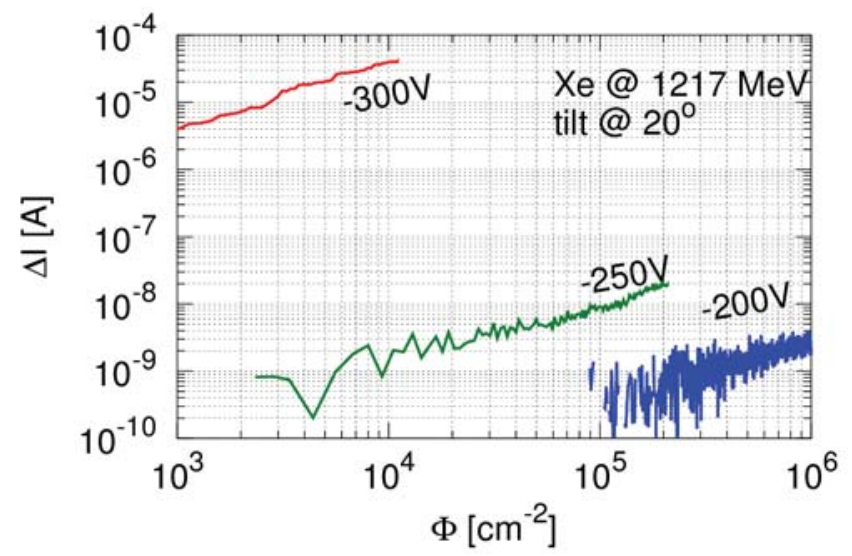

Figure 1. Change in leakage current for STPSC10H065DY diode as a function of cumulative $\mathrm{Xe}$ ion fluence at $20^{\circ}$ tilting angle for three different biasing configurations. All data are obtained for a single device.

The linear dependence of leakage current on the ion fluence is clearly visible. The rate of degradation is defined by the slope of these data curves. Both device types used in this work exhibited similar responses to the Xe-ion exposure.

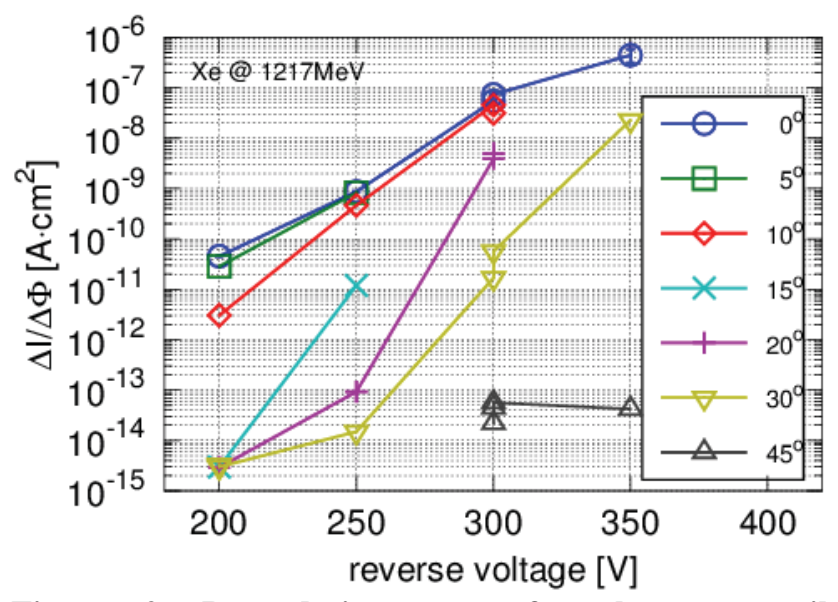

Figure 2. Degradation rates for the seven tilting configurations measured for STPSC10H065DY diodes as a function of bias voltage applied during irradiation for 1217-MeV Xe-ion exposure.

The degradation rates (leakage current/ion fluence) for an STPSC10H065DY diode at several tilting angles are presented in Figure 2 as a function of bias voltage applied during irradiation. Here it is clearly observed 
that the degradation rates, obtained for a tilted configuration, are lower than that for a normal incidence beam, especially at lower bias voltages. At higher voltages, the difference becomes smaller. Nevertheless, these data illustrate the fact that the degradation rate depends strongly on the bias voltage, as reported earlier in [15].

When the bias voltage is constant and the tilting angle increases, the rate of degradation decreases and it exhibits a dramatic decrease above a certain biasdependent angle. This effect is illustrated in Figure 3, where the evolution of leakage current for an STPSC10H065DY diode during Xe-ion exposure is given for several different tilting angles. For these data, the devices were biased at $-200 \mathrm{~V}$ during exposure.

Here the degradation rates at angles for $15^{\circ}$, and above, are below the detection limit of the equipment setup. Even though the leakage current for angles between $15^{\circ}$ and $30^{\circ}$ seems to increase during ion exposure, the current-voltage characteristics, determined after the exposure exhibited no significant change. The effect of shadowing from the bonding wire and the package edges, were visually checked at higher tilting angles, and can be considered not to cause the observed reduction in the degradation rates.

The strong angular dependence is summarized in Figure 4, where the degradation rates are given for both $\mathrm{SiC}$ diode types of this work, irradiated under bias voltages from $-200 \mathrm{~V}$ to $-350 \mathrm{~V}$, and at tilting angles ranging from $0^{\circ}$ to $45^{\circ}$. The data show clearly the strong angular dependence of the degradation rate. The degradation response is very symmetrical for positive and negative tilting angles. At a bias voltage of $-200 \mathrm{~V}$,

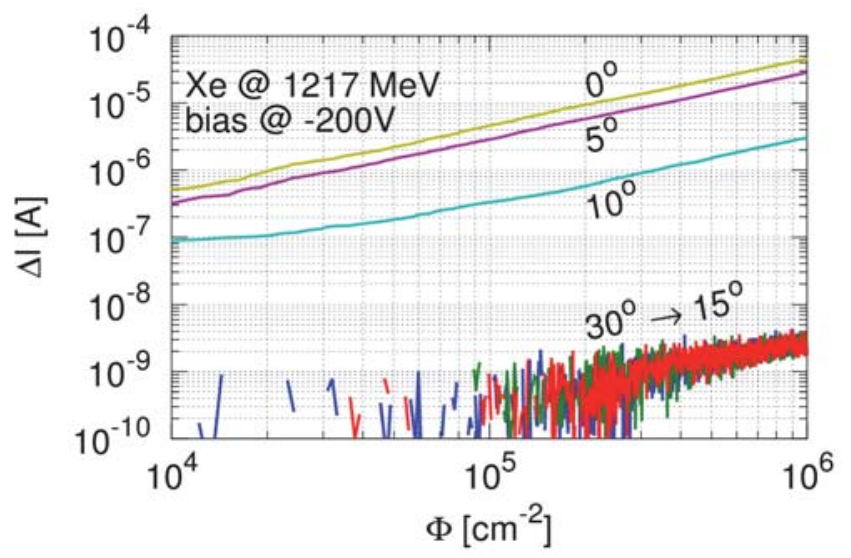

Figure 3. The change in leakage current for STPSC10H065DY diode as a function of cumulative ion fluence for 1217-MeV Xe ions at different tilting angles, obtained while the diode was biased at $-200 \mathrm{~V}$. All data are obtained for a single device. the degradation rate decreases below the detection limit already at $15^{\circ}$ of tilting, as illustrated in both Figure 3 and Figure 4. At higher bias voltage, the degradation still depends very strongly on the tilting angle, but higher tilting angles are required for reducing the degradation below the detection limit.
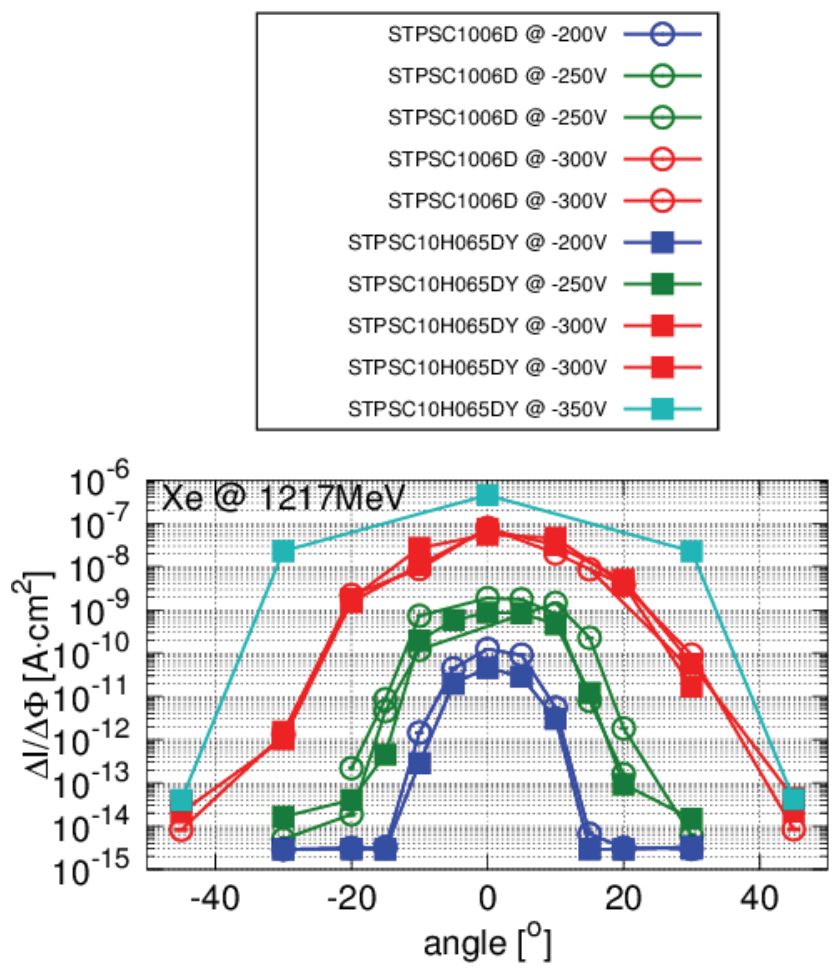

Figure 4. Degradation rate as a function of tilting angle measured by using bias voltages from $-200 \mathrm{~V}$ to $-350 \mathrm{~V}$ during irradiation for Xe ion exposure. Data for each bias voltage for each of the devices are taken for a single device.

The data in Figure 4 are presented as a heat map in Figure 5 that illustrates the increase in the solid angle of vulnerability for heavy-ion induced degradation for these SiC Schottky diodes. The degradation rate at $-350 \mathrm{~V}$ bias and normal incidence is almost four orders of magnitude greater than that at $-200 \mathrm{~V}$ bias. The degradation induced by $\mathrm{Xe}$-ions in these diodes at $-350 \mathrm{~V}$ is reduced below the detection limit at a tilting angle of approximately $45^{\circ}$, whereas for $-200 \mathrm{~V}$ this occurs already at angles $\sim 15^{\circ}$. These kinds of data could be used in estimating the on-orbit behavior of these types of parts. However, in order to obtain more precise degradation estimates, experimental data for other ions (lighter than $\mathrm{Xe}$ ) would be required. 


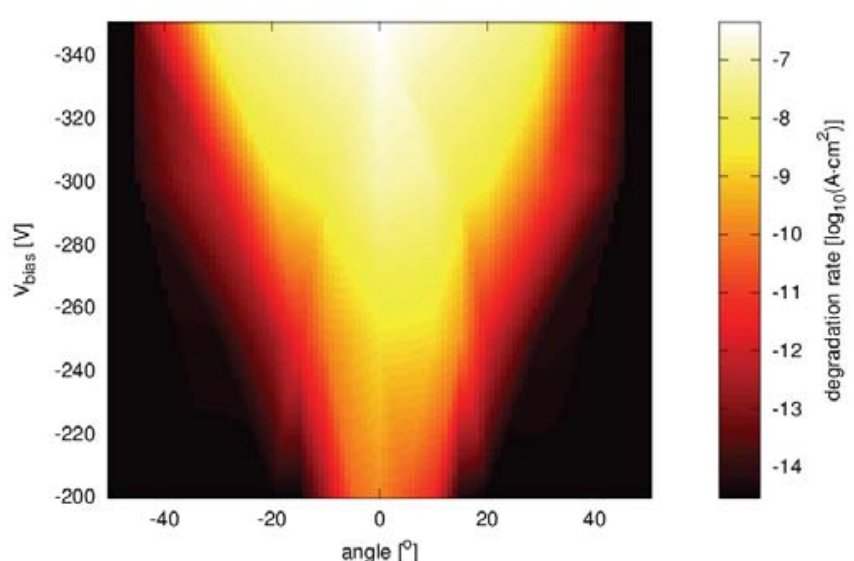

Figure 5. The heat map of the degradation rate as functions of tilting angle (abscissa) and the bias voltage (ordinate) obtained for 1217-MeV Xe-ion beam. Black color corresponds to the area, where no degradation is observed.

\section{TCAD SIMULATIONS AND DISCUSSION}

In order to develop insight into the heavy-ion induced thermo-electric response in the diode structure, VICTORY DEVICE TCAD tools from Silvaco Inc. [16] have been used. In the simulations, a 3D model was used with lateral dimensions of $40 \mu \mathrm{m} \times 40 \mu \mathrm{m}$ for a normal ion strike, and $40 \mu \mathrm{m} \times 80 \mu \mathrm{m}$ for tilted ion tracks (the ion track Gaussian radius was $0.05 \mu \mathrm{m}$ ). The vertical depth of the 3D model was $120 \mu m$, which was verified not to affect the temperature results with respect to the full depth of the actual experimental $\mathrm{SiC}$ substrate depth $(\sim 300 \mu \mathrm{m})$. Note that the range of the Xe ions at 1217 $\mathrm{MeV}$ in $\mathrm{SiC}$ is about $60 \mu \mathrm{m}$. For the average electronhole pair creation energy in $\mathrm{SiC}$, a value of $7.5 \mathrm{eV}$ was used, which is an average between the recently published experimental values of $7.28 \mathrm{eV}$ [17] and $7.8 \mathrm{eV}[18]$. For modeling the avalanche multiplication, an anisotropic impact ionization model that is especially developed and calibrated for $4 \mathrm{H}-\mathrm{SiC}$ power devices [19] has been implemented in Silvaco TCAD tools, and was used in this work. Also, the thermionic emission model was activated in all simulations.

Figure 6 shows the temporal evolution of the anode current in the SiC Schottky diode simulated for Xe-ion strikes at normal incidence and at tilting angles of $3^{\circ}$, $5^{\circ}, 10^{\circ}, 15^{\circ}$ and $30^{\circ}$. In the simulations, the charge, representing the ion strike, is deposited with a Gaussian time profile that has a peak at time $t=5 \mathrm{ps}$ and a rise time of $0.5 \mathrm{ps}$. The anode current reaches its maximum at about $t=6.3 \mathrm{ps}$ at all simulated tilting angles. Moreover, the charge deposited by the ion modifies the electric field within the Schottky structure. This is illustrated in Figure 7, where the simulated electric field distribution along the center of the ion track is presented before, and at two time instants after the ion strike.

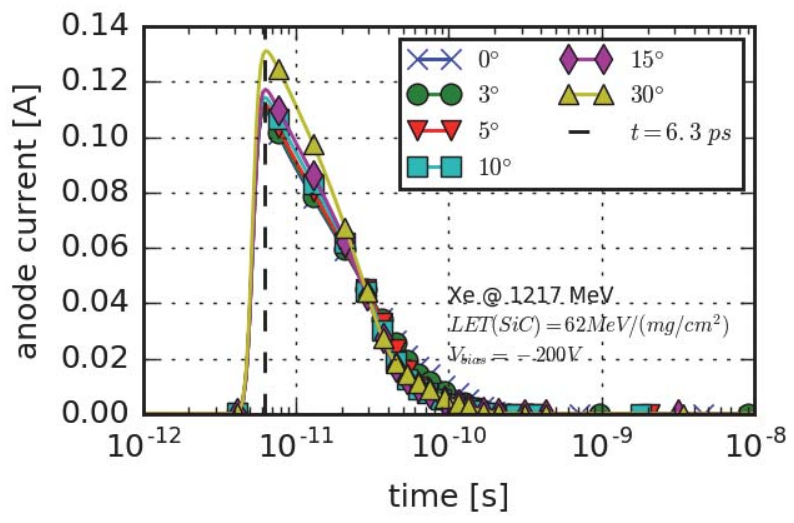

Figure 6. Temporal evolution of the anode current. Note: The data for $0^{\circ}$ angle lies behind the other data plots. The current data for $0^{\circ}$ and $3^{\circ}$ are almost identical.

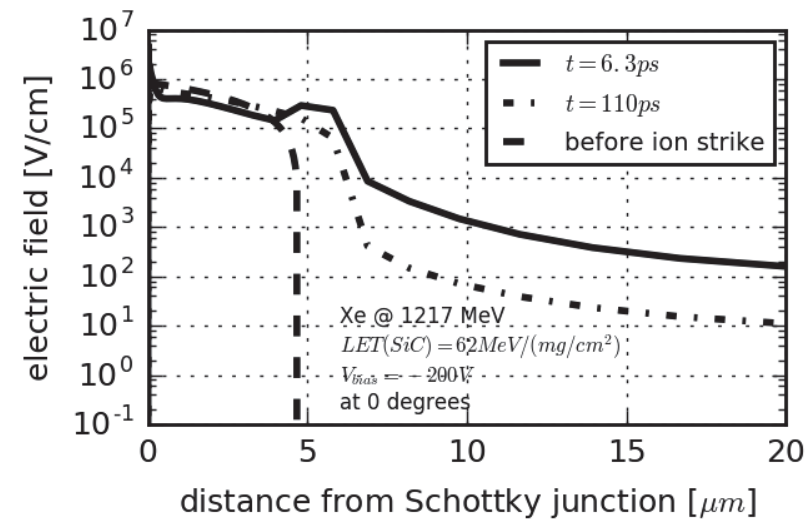

Figure 7. Electric field distribution along the center of the ion track before (blue dashed), and at time instants of $6.3 p s$ (solid) and $110 p s$ (dash-dot). These data correspond to $-200 \mathrm{~V}$ bias voltage and normal ion incidence.

In addition, the heat power density at the ion hit location just underneath (1 $\mathrm{nm}$ below) the Schottky junction was determined. The heat power density is defined by the scalar product of current density and electric field, that will cause temperatures to rise in the structure via Joule heating. The results are presented in Figure 8. From these data, one can see that the heat power density exhibits two maxima at this location, one at $t \approx 6.3 p s$ and another at $t \approx 110 p s$. A close analysis of the 3D TCAD simulation results suggests that the carriers (electrons and holes) at the ion track boundaries diffuse much faster than the ones from the track core (due to high gradients at the track side boundaries), so after a while ( $100 \mathrm{ps})$ the highly-conductive path (i.e. track core) becomes even narrower, so the current 
density in the core becomes even higher for a moment, due to "squeezing" of the total current into this narrower conductive path. This leads to a momentary increase of power density in the second peak. The figure also shows the lattice temperature at $5 \mathrm{~nm}$ below the Schottky junction. Evidently, the lattice temperature reaches its maximum when the heat power density reaches its second maximum. However, both peaks in the heat power density play a role in the total lattice temperature evolution. Because there is no significant difference in the electric field before and after the ion strike at this location, the temperature rise can be attributed to the changes in the current density.

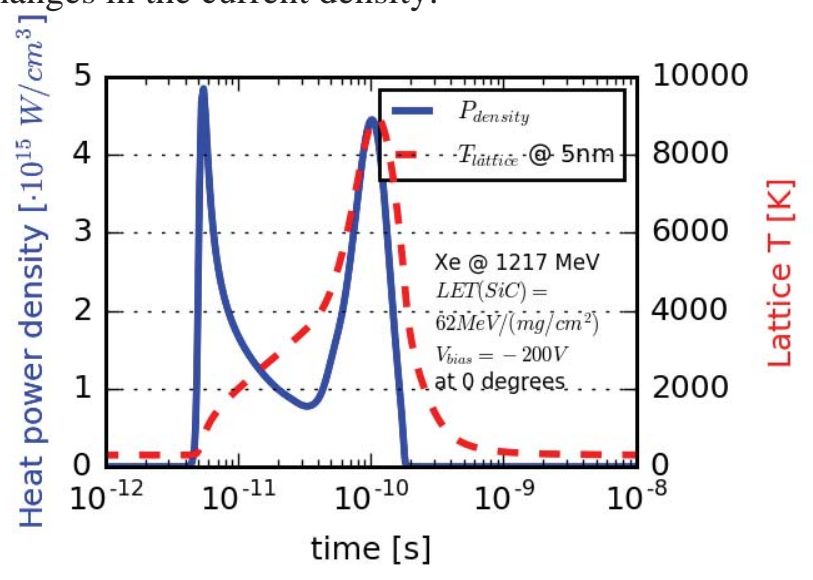

Figure 8. The heat power density (solid blue) and the SiC lattice temperature (dashed red) at the ion hit location just $5 \mathrm{~nm}$ below the Schottky junction for $-200 \mathrm{~V}$ bias and normal ion incidence.

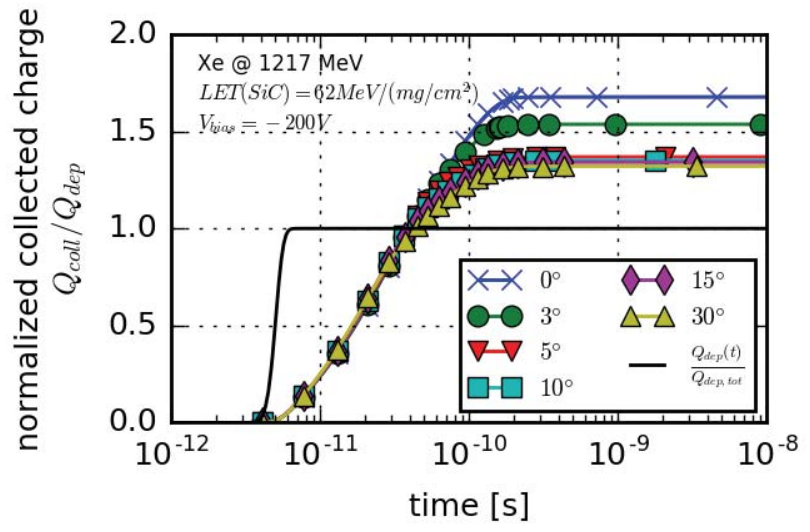

Figure 9. Temporal evolution of the charge collection in $\mathrm{SiC}$ Schottky structure, biased at $\mathbf{- 2 0 0 V}$ and hit by Xe-ion. The collected charge $Q_{\text {coll }}$ is normalized with the total deposited charge $Q_{d e p}$ in the depletion region. Also, the charge deposition timing is illustrated (solid black line).

The electron-phonon coupling can be assumed to occur at timescales of < 1ps [20], [21]. This means that during the Joule heating, the temperature equilibrium between electrons and the lattice atoms is achieved faster than the timescales involved in the charge collection. Time scales for cumulative charge deposition and collection for different tilting configurations are presented in Figure 9. Figure 10 presents the corresponding simulated temporal evolution of the maximum lattice temperatures. In these simulations, $-200 \mathrm{~V}$ bias was applied to the diode corresponding to one of the experimental configurations discussed above. The results show dramatic differences in the maximum local temperatures occurring in a reverse-biased diode structure promptly after the ion strike at different ion incident angles. The simulations show that the maximum temperature is obtained at or near the Schottky contact, along the path of the incident ion. For normal incidence, the size of the volume where the temperature exceeds the melting temperature of $\mathrm{SiC}(3000 K)[22]$ is estimated to be about $80 \mathrm{~nm}$ across and $100 \mathrm{~nm}$ along the track. The location for the maximum peak SiC lattice temperature is reached few nanometers below the Schottky junction and it is reached at about 110 ps after the ion strike.

In the simulation results given in Figure 10, already at $5^{o}$ the maximum lattice temperature in the structure is much less than the values obtained at the normal incidence. Moreover, at higher tilting angles the maximum lattice temperatures are even lower. This is attributed to fact that since the current density and electric field vectors are aligned they produce higher power densities than at tilted configuration.

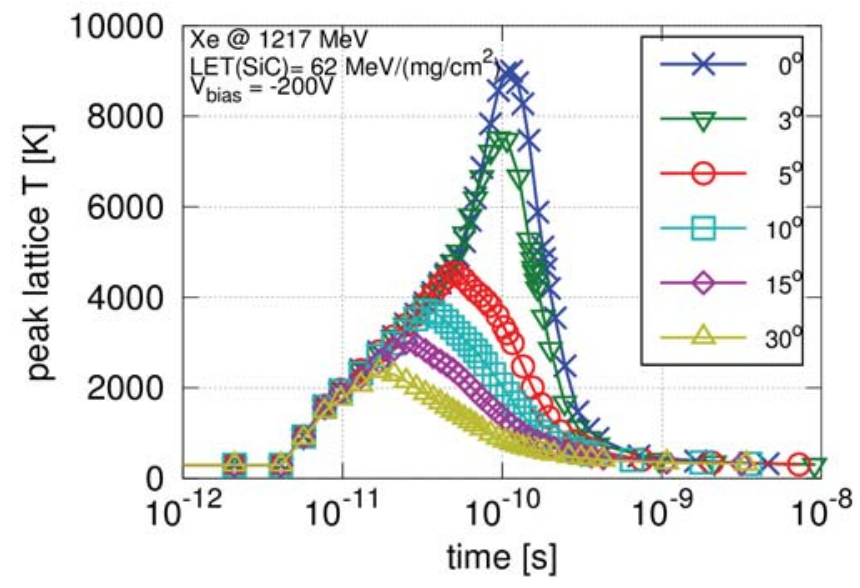

Figure 10. Temporal evolution of the maximum lattice temperatures at six different ion incident angles, simulated in SiC Schottky diode structure after 1217-MeV Xe ion strike. The average LET value of $62 \mathrm{MeV} /\left(\mathrm{mg} / \mathrm{cm}^{2}\right)$ is used for estimating the ion's energy deposition. After [11].

For an ion strike at normal incidence, the simulated maximum lattice temperature values exceed $9000 \mathrm{~K}$, which is much higher than the melting temperature of 
SiC. These simulation results are only qualitative, as the models used in TCAD are not verified at these high temperatures. E.g., the values for electron and hole mobilities in $\mathrm{SiC}$ are experimentally validated only for temperatures up to about $700 \mathrm{~K}$ [23]. Above this temperature the parameter values used in the simulations are based on extrapolations. Most importantly, these temperature values should be used only as a proxy indicator for the assumed damage in the lattice; i.e., when the simulations indicate that the lattice temperature approaches or exceeds the melting temperature, there is a high likelihood of damage, but the specific temperature reached is not known. Even though the temperature values obtained from TCAD simulations do not present a precise measure of the local lattice properties at very short timescales and small volumes, the simulations indicate that at normal incidence the energy dissipation is much higher than in the tilted configuration.

Hence, the maximum energy dissipation occurring at normal incidence appears to be sufficient to cause material modifications that induce higher leakage current degradation in SiC Schottky diodes under heavy-ion exposure compared to that at tilted beam angles.

The decreased energy dissipation with increasing tilting angle is consistent with the lower degradation rates. Moreover, the lower energy dissipation at higher angles can be explained by the misalignment of the heavy-ion induced charge filament and the potential gradient within the depletion region. This reduces the current density within the Schottky structure, which leads to reduced density of overall energy dissipation. This reduction in current density can also be estimated with simple geometrical analysis of the ion track. The ion's trajectory within the depletion layer is illustrated in Figure 11.

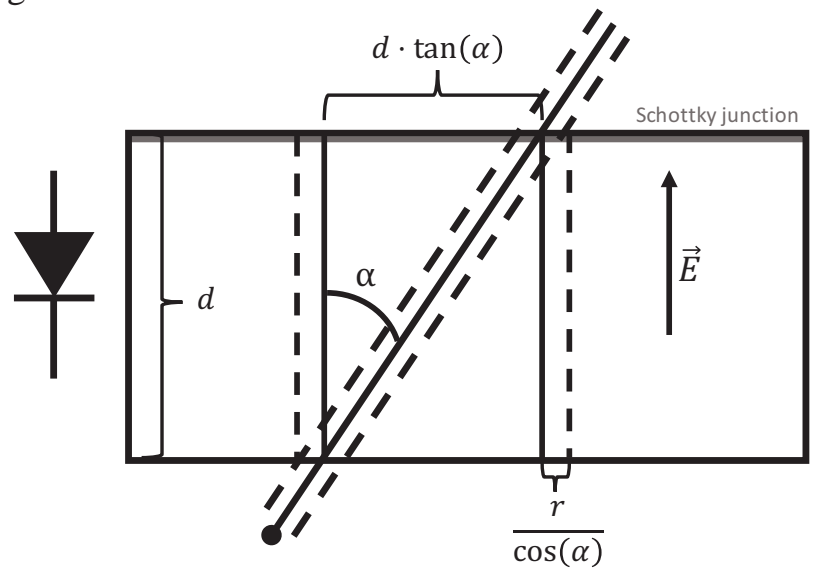

Figure 11. Illustration of the ion passing through the depletion layer at a tilting angle $\alpha$.

With a similar analysis as presented in [8], we have assumed that the ion deposits its energy within a certain radius, $r$, from its trajectory. Hence, the initial iondeposited energy in the depletion region can be estimated to be within a volume of $V_{d e p}=\pi \cdot r^{2}$. $d / \cos (\alpha)$, where $d$ is the bias-dependent thickness of the depletion layer and $\alpha$ is the tilt angle. One should keep in mind that in reality the ion-deposited energy within the ion track is highly concentrated near the ion's trajectory [24], but for simplicity here an even energy distribution is assumed within a given track radius $r$.

The deposited energy is now assumed to be transformed fully into charge, not considering possible recombination or charge multiplication effects at this stage. Moreover, this charge is collected by the electric field that is normal to the surface of the diode. Now, we can consider the charge collection volume, from which the charge is collected, to increase with increasing angle. The general form for the charge collection volume can be written

$$
V_{\text {coll }}=\left(\frac{\pi r^{2} d}{\cos (\alpha)}+2 r d^{2} \tan (\alpha)\right)
$$

We also consider the area where the charge collection occurs at the anode. At normal incidence, the area is simply $A_{0}=\pi r^{2}$. At tilted angles the collected charge is projected to the anode within an area of

$$
A_{\text {coll }}=\left(\frac{\pi r^{2}}{\cos (\alpha)}+2 r d \tan (\alpha)\right)
$$

or simply, $A_{\text {coll }}=\frac{V_{\text {coll }}}{d}$. The charge collection area basically determines the current density and therefore the power density due to Joule heating.

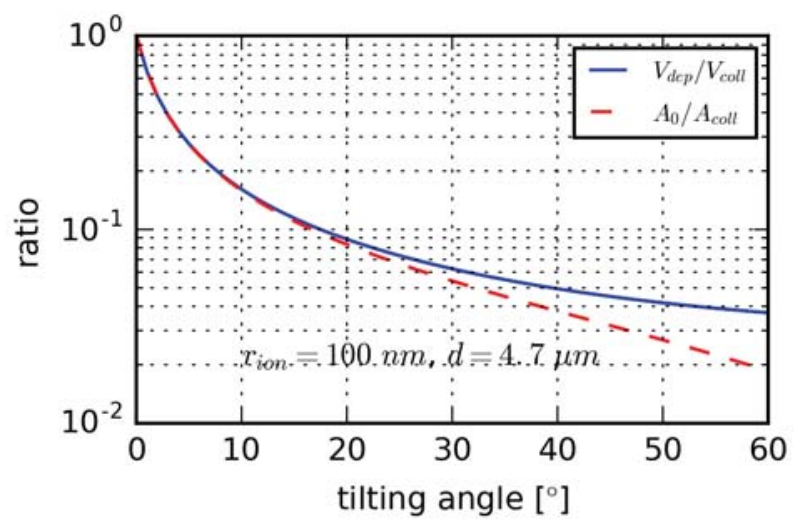

Figure 12. Ratio between the charge deposition and collection volumes (solid blue), and between the charge collection areas at normal incidence and at tilted angles (dashed red).

By looking at the ratios of both $\frac{V_{\text {dep }}}{V_{\text {coll }}}$ and $\frac{A_{0}}{A_{\text {coll }}}$, we can see a very clear reduction as a function of tilting angle. 
This is illustrated in Figure 12. For these data, we have used values $r=100 \mathrm{~nm}$ and $d=4.7 \mu \mathrm{m}$. The used depletion layer thickness of $4.7 \mu \mathrm{m}$ used in these calculations corresponds to the value for $-200 \mathrm{~V}$ bias.

From the TCAD simulation data one can obtain the collected charge, $Q_{\text {coll }}$, after the ion strike. By comparing this to the charge, $Q_{\text {dep }}$, deposited by the ion within the depletion region, the avalanche multiplication due to high electric field can be estimated.

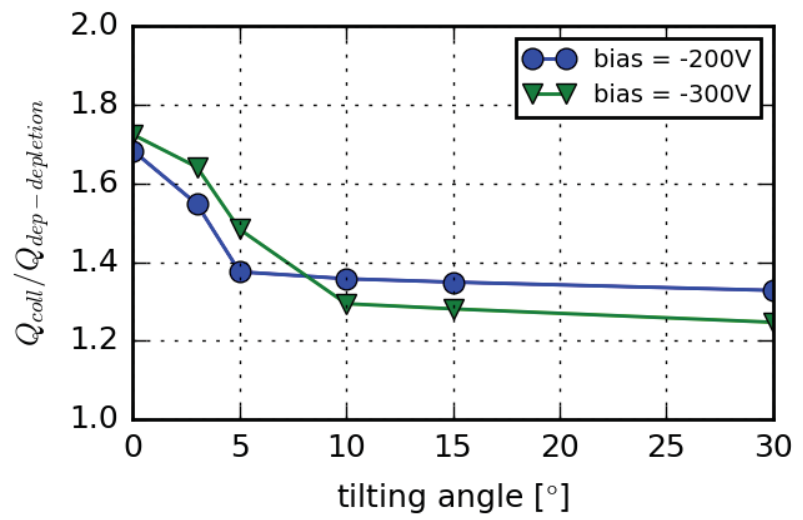

Figure 13. Ratio of the total collected charge to the total deposited charge in the depletion region for the simulated SiC Schottky diode.

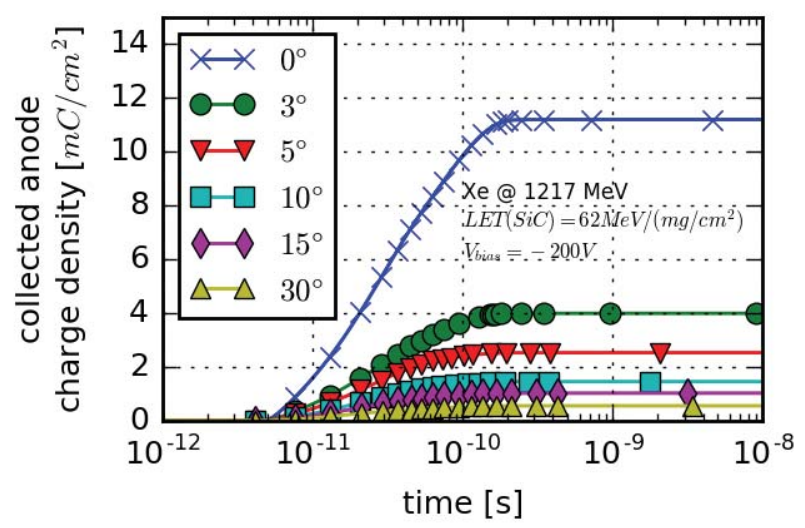

Figure 14. Temporal evolution of the collected charge density at the anode for different tilt angles and for $-200 \mathrm{~V}$ bias voltage. These data correspond to the charge collection and temperature data presented in Figure 9 and Figure 10, respectively.

The ratio between collected charge and deposited charge is presented in Figure 13 as a function of tilting angle for two biasing conditions. These data show a reduction in charge multiplication with increasing tilt angle. Over all, the charge multiplication ranges between 1.2 to 1.7 for both considered bias voltages. The difference between the charge (avalanche) multiplication at normal incidence and at tilted angles is not sufficient to alone explain the experimental observations.

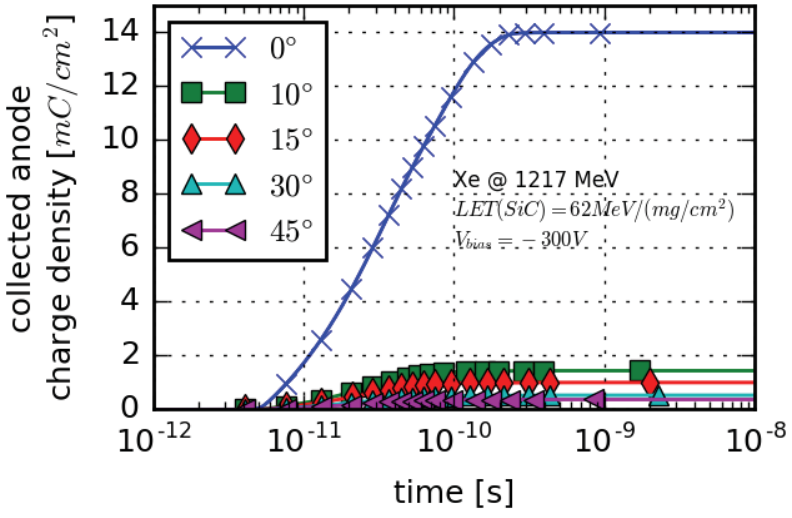

Figure 15. Temporal evolution of the collected charge density at the anode for different tilt angles and for $\mathbf{- 3 0 0 \mathrm { V }}$ bias voltage.

However, by integrating the simulated charge that is accumulated at the anode after the ion strike, and by estimating from Eq.(2) the area where the charge is collected, we can illustrate the effect of tilting on the collected charge density. These data are presented in Figure 14 and Figure 15, where the cumulative charge collected at the anode is plotted as a function of time after the ion strike for several tilting angles and for two different bias configurations.

The simulation results in Figure 14 correspond to the charge collection and temperature data presented in Figure 9 and Figure 10, respectively. These calculations assume the collected charge is evenly distributed over the whole area given by Eq.(2). In reality, the process is more complex and the details of the charge collection are different, but this back-of-the-envelope calculation provides insight about the underlying phenomenon.

All in all, from these data, we can see a dramatic reduction in the density of collected charge at the anode (i.e., through the Schottky junction) with increasing tilt angle. This is assumed to cause the observed reduction in the degradation rates. The total cumulative charge, collected at normal incidence and at higher bias, is attributed to higher deposited charge due to thicker depletion region.

As mentioned above, the dissipated power density that governs the Joule heating, depends on the inner product of the electric field and the current density. Hence, at higher angles the reduced collected charge density will result in reduced power densities, which in turn can be assumed to cause the experimentally observed reduction in the ion-induced degradation rates in the SiC Schottky diodes.

At normal incidence, the higher power density at higher reverse bias explains also the higher degradation rates observed at higher reverse biases. 


\section{CONCLUSIONS}

This work presents the dependence of heavy-ion induced degradation in SiC Schottky power diodes on the tilting angle. The degradation is observed to be the highest at normal incidence. Dramatic reduction in the degradation rates are observed at beam tilting angles at and above about $10^{\circ}$ with respect to normal of the device surface, depending on the applied reverse bias. At higher reverse bias voltages, higher tilting angles are required in order to reduce the degradation below the detection limit.

TCAD simulations indicate that this observation could be explained by the differences in the prompt thermal effects within the SiC Schottky structure at different tilting angles. A thermal spike within the $\mathrm{SiC}$ lattice is generated by the synergetic effects of applied reversebias and the heavy-ion strike. When an ion traverses the Schottky diode structure at angles that are not parallel to the applied electric field, the maximum simulated lattice temperatures are reduced dramatically. Schematically this can be explained by the geometry of the ion-induced charge deposition. At higher tilting angles the induced charge is collected within a larger area compared to the area at normal incidence. Hence the power density, determined by the inner product of the electric field and current density vectors, is decreased with increasing tilting angle. This decrease in the power density is assumed to cause the experimentally observed reduction in the ion-induced degradation rates in the SiC Schottky diodes at higher tilting angles.

The results presented in this work can be used for improving the error rate and survivability estimations for SiC Schottky power devices operating in harsh radiation environments. However, more experimental data are needed for ions lighter than Xe, which was used in this work.

\section{ACKNOWLEDGMENT}

The authors would like to thank Silvaco Inc. for their support. Also, the help from Professors Dan Fleetwood and Sokrates Pantelides of Vanderbilt University, and Dr. Heikki Kettunen, Mikko Rossi and Jukka Jaatinen of the University of Jyväskylä is gratefully acknowledged.

\section{REFERENCES}

S. Kuboyama, C. Kamezawa, Y. Satoh, T. Hirao, and H. Ohyama, "Single-Event Burnout of Silicon Carbide Schottky Barrier Diodes Caused by High Energy Protons," IEEE Trans. Nucl. Sci., vol. 54, no. 6, pp. 2379-2383, Dec. 2007.

[2] E. Mizuta, S. Kuboyama, H. Abe, Y. Iwata, and T. Tamura, "Investigation of Single-Event Damages on Silicon Carbide ( $\mathrm{SiC})$ Power MOSFETs," IEEE Trans. Nucl. Sci., vol. 61, no. 4, pp. 1924 1928, Aug. 2014.
[3] L. Scheick, L. Selva, and H. Becker, "Displacement damageinduced catastrophic second breakdown in silicon carbide Schottky power diodes," IEEE Trans. Nucl. Sci., vol. 51, no. 6, pp. 3193 3200, Dec. 2004.

[4] M. Allenspach et al., "SEGR and SEB in n-channel power MOSFETs," IEEE Trans. Nucl. Sci., vol. 43, no. 6, pp. 2927-2931, 1996.

[5] V. Ferlet-Cavrois et al., "Influence of Beam Conditions and Energy for SEE Testing," IEEE Trans. Nucl. Sci., vol. 59, no. 4, pp. 11491160, Aug. 2012.

[6] S. Kuboyama, C. Kamezawa, N. Ikeda, T. Hirao, and H. Ohyama, "Anomalous Charge Collection in Silicon Carbide Schottky Barrier Diodes and Resulting Permanent Damage and Single-Event Burnout," IEEE Trans. Nucl. Sci., vol. 53, no. 6, pp. 3343-3348, Dec. 2006.

[7] A. Javanainen et al., "Charge Transport Mechanisms in Heavy-Ion Driven Leakage Current in Silicon Carbide Schottky Power Diodes," IEEE Trans. Device Mater. Reliab., vol. 16, no. 2, pp. 208-212, Jun. 2016.

[8] I. Mouret, M. Allenspach, R. D. Schrimpf, J. R. Brews, K. F. Galloway, and P. Calvel, "Temperature and angular dependence of substrate response in SEGR [power MOSFET]," IEEE Trans. Nucl. Sci., vol. 41, no. 6, pp. 2216-2221, Dec. 1994.

[9] C. Abbate et al., "Analysis of Heavy Ion Irradiation Induced Thermal Damage in SiC Schottky Diodes," IEEE Trans. Nucl. Sci., vol. 62, no. 1, pp. 202-209, Feb. 2015.

[10] C. Abbate et al., "Thermal damage in SiC Schottky diodes induced by SE heavy ions," Microelectron. Reliab., vol. 54, no. 9-10, pp. 2200-2206, Sep. 2014.

[11] A. Javanainen et al., "Incident Angle Effect on Heavy Ion Induced Reverse Leakage Current in SiC Schottky Diodes," in Proc. 2016 16th European Conference on Radiation and Its Effects on Components and Systems (RADECS), 2016, pp. 1-4.

[12] A. Virtanen, H. Kettunen, A. Javanainen, M. Rossi, and J. Jaatinen, "RADiation Effects Facility at JYFL." [Online]. Available: http://www.jyu.fi/accelerator/radef.

[13] A. Javanainen, "European Component Irradiation Facilities Cocktail Calculator," 2015. [Online]. Available:

http://research.jyu.fi/radef/ECIFcalc/.

[14] Keithley/Tektronix, "Keithley 2400 Series," Keithley Datasheets. [Online]. Available:

http://www.tek.com/sites/tek.com/files/media/media/resources/Ser2 400.pdf.

[15] A. Javanainen et al., "Heavy Ion Induced Degradation in $\mathrm{SiC}$ Schottky Diodes: Bias and Energy Deposition Dependence," IEEE Trans. Nucl. Sci., vol. 64, no. 1, pp. 415-420, Jan. 2017.

[16] Silvaco Inc., Victory Device User's Manual. Santa Clara, CA, USA, 2016.

[17] S. K. Chaudhuri, K. J. Zavalla, and K. C. Mandal, "Experimental determination of electron-hole pair creation energy in $4 \mathrm{H}-\mathrm{SiC}$ epitaxial layer: An absolute calibration approach," Appl. Phys. Lett., vol. 102, no. 3, p. 31109, Jan. 2013.

[18] T. R. Garcia, A. Kumar, B. Reinke, T. E. Blue, and W. Windl, "Electron-hole pair generation in $\mathrm{SiC}$ high-temperature alpha particle detectors," Appl. Phys. Lett., vol. 103, no. 15, p. 152108, Oct. 2013.

[19] T. Hatakeyama, J. Nishio, C. Ota, and T. Shinohe, "Physical Modeling and Scaling Properties of $4 \mathrm{H}-\mathrm{SiC}$ Power Devices," in 2005 International Conference On Simulation of Semiconductor Processes and Devices, 2005, pp. 171-174.

[20] M. Backman et al., "Molecular dynamics simulations of swift heavy ion induced defect recovery in SiC," Comput. Mater. Sci., vol. 67, pp. 261-265, Feb. 2013.

[21] O. Ochedowski et al., "Graphitic nanostripes in silicon carbide surfaces created by swift heavy ion irradiation," Nat. Commun., vol. 5, p. 16102, Jun. 2014.

[22] C. Buttay, C. Raynaud, H. Morel, G. Civrac, M.-L. Locatelli, and F. Morel, "Thermal Stability of Silicon Carbide Power Diodes," IEEE Trans. Electron Devices, vol. 59, no. 3, pp. 761-769, Mar. 2012.

[23] M. Roschke and F. Schwierz, "Electron mobility models for $4 \mathrm{H}$, 6H, and 3C SiC [MESFETs]," IEEE Trans. Electron Devices, vol. 48, no. 7, pp. 1442-1447, Jul. 2001.

[24] R. Katz, "Track structure theory in radiobiology and in radiation detection," Nucl. Track Detect., vol. 2, no. 1, pp. 1-28, 1978. 